\title{
Cantwell-Ransley technique in complete epispadias repair
}

\author{
Khaled A. Ismail, MD; Mohamed H. Mazhar Ashour, MD; \\ Akram El Batarny, MD; Mohamed Hashish, MD
}

Pediatric Surgery Unit-Department of General Surgery-Tanta University.

Background/Purpose: Epispadias is a rare congenital anomaly of the external genitalia occurring in approximately one in 118000 males and one in 400000 females. The purpose of this study is to evaluate the results of Cantwell-Ransley operation in the repair of complete epispadias.

Patients \& methods: Between June 2006 and June 2012, 11 patients with complete epispadias were treated with the Cantwell-Ransley technique at the Pediatric Surgery UnitTanta University Hospital. Patients were followed up for 3 months to report any complication.

Results: 11 male cases with epispadias were admitted to the Pediatric Surgery Unit-Tanta University Hospital in the period from June 2006 to June 2012 with history of bladder exstrophy repair 1-2 years earlier. All cases were peno-pubic. Their age ranged from 1 7/12 years to 3 years, with a mean of $23 / 12$ years. Cantwell-Ransley operation was performed to all patients with satisfactory results. Only one case showed mild wound infection that was conservatively managed. Another case developed urethro cutaneous fistula, which was surgically treated by simple excision \& closure after 6 months of epispadias repair.

Conclusion: The cosmetic and functional outcomes of Cantwell-Ransley epispadias repair appear to be excellent.

Key words: Epispadias, Cantwell-Ransley operation.

\section{Introduction:}

Epispadias is a rare congenital anomaly of the external genitalia occurring in approximately one in 118000 males and one in 400000 females. ${ }^{1}$ The defect however is also seen in association with classic bladder exstrophy (the exstrophy-epispadias complex) with an incidence of approximately one in $40000 .^{2}$ The first documented report of epispadias dates back to AD 610-641 and Byzantine Emperor Heraclius. ${ }^{1}$ According to meatal position certain degrees of severity have been described, including balanic epispadias (the less severe and less common grade), penile or continent epispadias (some degree of incontinence is often present) and peno-pubic or incontinent epispadias. In the latter category the whole urethral plate is widely open as well as the bladder neck, the external sphincter is deficient, the pubic bones are separated to various degrees, and the penis is short and connected to the pubis. ${ }^{3}$

In 1895, Cantwell introduced the true urethroplasty for reconstruction of male epispadias. He created a tube from the urethral plate, freed it completely to its proximal base and then transplanted it below the corpora cavernosa. The technique was modified in 1903 by Bullitt who, after losing most of the urethral plate, inadvertently discovered that the preputial skin could be used for the terminal segment. For most of the last 50 years the most popular technique used for epispadias repair has been the Young modification of Cantwell technique. $^{3}$ Gross and Cresson mobilized the urethral plate from both corpora but with a narrow attachment to the ventral penile skin for blood supply. McIndow converted epispadias to hypospadias by tabularizing the urethral plate and transposing it ventrally beneath the corpora. In 1963, Michalowski and Modelski recommended a multi-stage 
epispadias repair. Since then, urethral repairs using preputial graft or bladder mucosa graft have been described.4-9 The wide variety of techniques reflects the generally unsatisfactory cosmetic and functional results from penile reconstruction. In 1989, Ransley et $\mathrm{al}^{3}$ reported a modification of the earlier Cantwell repair. Since then, due to the excellent cosmetic and functional results achieved with this technique, the CantwellRansley epispadias repair has been widely adopted. ${ }^{3}$

\section{Patients and methods:}

Between June 2006 and June 2012, 11 patients with complete epispadias were treated with the Cantwell-Ransley technique at the Pediatric Surgery Unit -Tanta University Hospital.

All cases were subjected to:

- Complete history taking.

- Thorough clinical examination.

- Complete urine analysis before operation, to detect and manage any urinary tract infection before surgery.

- Operative repair by using the Cantwell-Ransley technique.

Pre operative preparation often included treatment with testosterone to improve the vascularity of the preputial and penile skin and to increase the size of the penis. Patients received 3 intra muscular injections of $2 \mathrm{mg} / \mathrm{kg}$ testosterone at monthly intervals preoperatively. Also topical testosterone cream 5\% was applied to the urethral plate.

With the patient lying supine, artificial erection was performed by saline injection at both corpora cavernosa to detect presence of chordee and mark its site and extent. A traction suture was placed through the ventral aspect of the glans penis, and then a wide strip of urethral mucosa extending for the prostatic urethral meatus to the tip of the glans was outlined and incised on the dorsum. Thick glandular flaps were constructed bilaterally. The ventral skin was taken down to the level of the scrotum. Care was taken to preserve the vascularity of the urethral plate, which arised proximally and extended upward between the corpora as a blood supply to the urethral plate. The corpora were dissected ventrally on the surface of Buck fascia. The plane was followed closely bilaterally to the dorsum of the penis between the corpus spongiosum and the corporal body. The suspensory ligament was divided in some cases with small sized penis. The urethral plate was dissected just on the corporal bodies to the level of the prostate and the glans, respectively. Care was taken to leave the most distal one centimeter attachment of the mucosal plate to the glans intact. The urethral strip was tabularized over an 8 French silicon stent, and a tube was fashioned by continuous 5/0 PDS sutures.

Afterward, the corporal bodies were closed over the neo urethra. The now ventrally placed urethra was secured in place between the corpora. The glanular wings were closed and the ventral skin sutured to the ventral edge of the corona, while the flaps provided coverage of the dorsum.

A silicon stent was secured and a plastic occlusive dressing applied.

Patients were followed up for 3 months to report any complication.

\section{Results:}

Our study included 11 cases with epispadias, admitted to the Pediatric Surgery Unit-Tanta University Hospital in the period from June 2006 to June 2012. All cases were males, with history of bladder exstrophy repair 1-2 years earlier. All cases were penopubic type. Their age ranged from $17 / 12$ years to 3 years, with a mean of $23 / 12$ years.

2 cases had a history of associated bilateral congenital inguinal hernias, which were repaired in the same session after bladder closure.

Testosterone was given in a dose of $2 \mathrm{mg} /$ $\mathrm{Kg}$, in 3 monthly doses before operation, in addition to topical 5\% ointment applied to the urethral plate.

Cantwell-Ransley operation was performed to all patients as previously mentioned. Artificial erection by saline injection in both corpora was performed in the beginning of the procedure to detect presence or absence of dorsal chordee, which was seen in 3 cases. The site and extent was 


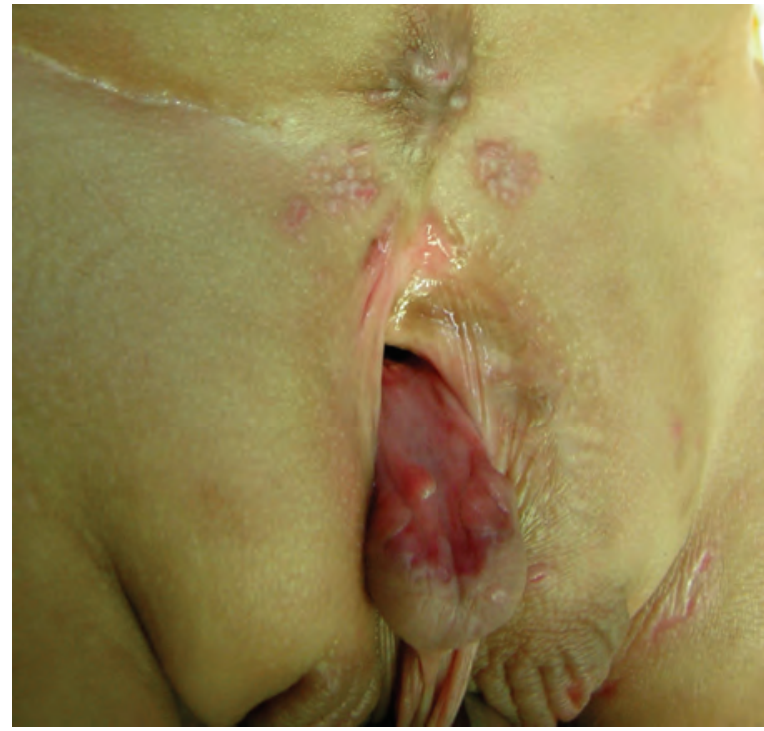

Figure (1): Peno-pubic epispadias after repair of bladder exstrophy.

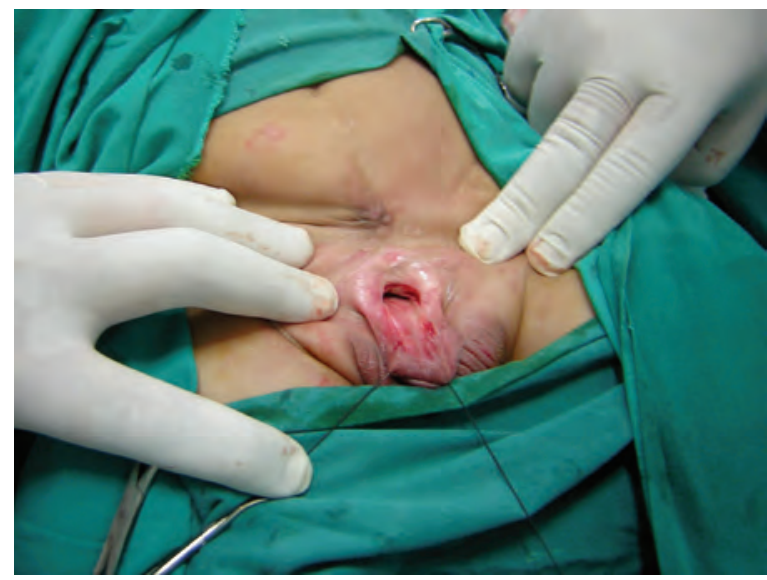

Figure (3): Traction sutures applied to Glans.

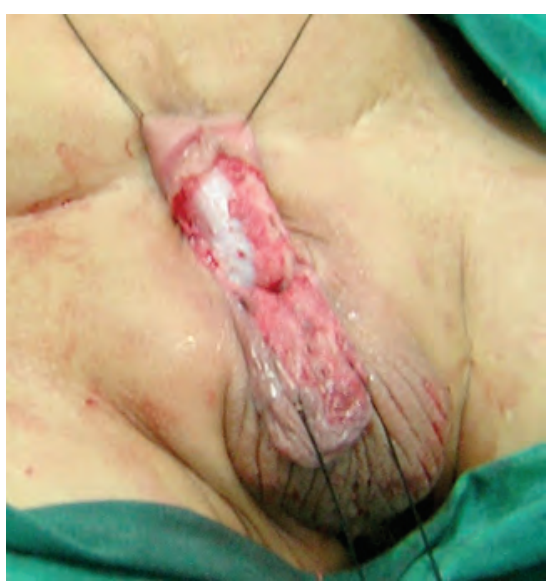

Figure (5): Degloving of Penile Skin.

determined. Fibrous tissue in the corpora responsible for chordee was excised, and artificial erection was performed again to deal with any residual chordee present.

Complete disassembly of suspensory

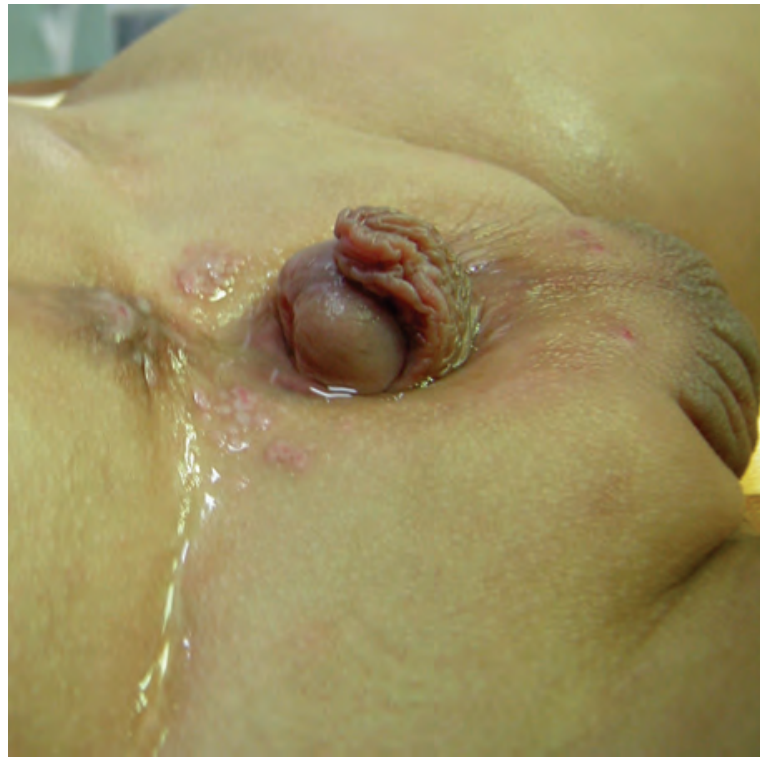

Figure (2): Ventral prepuce.

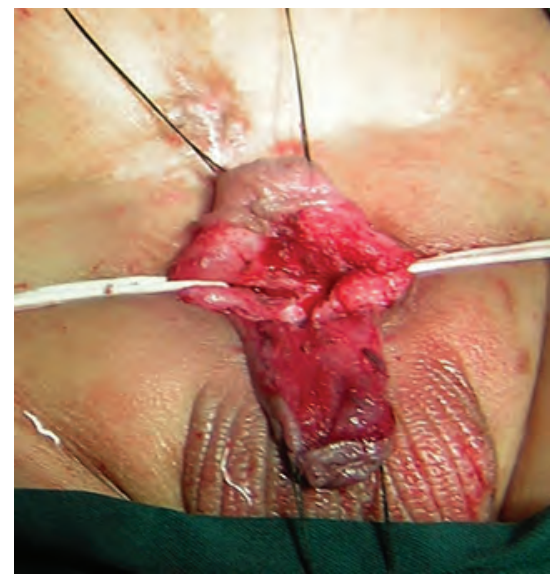

Figure (4): Marking the urethral flap.

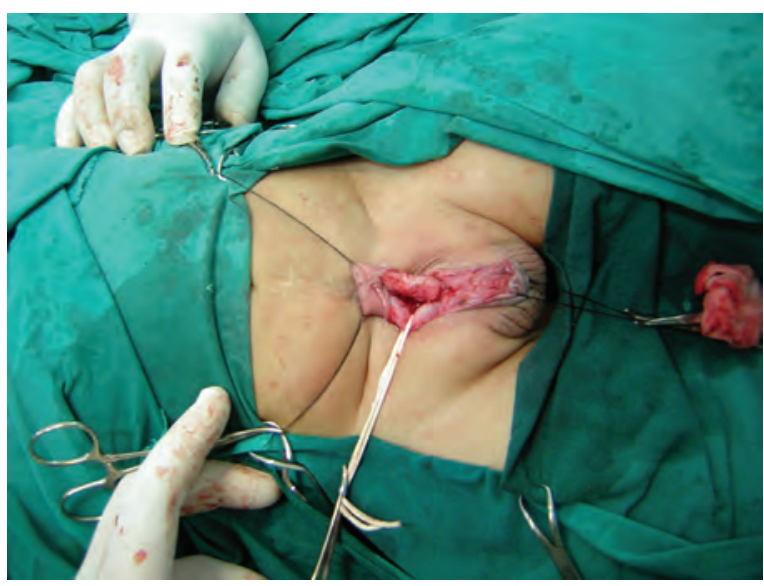

Figure (6): Dissection of corpora cavernosa.

ligament of the penis was resorted to only 5 cases, while the remaining cases underwent disassembly of both corpora without the suspensory ligament.

The urethral plate was dissected till its 


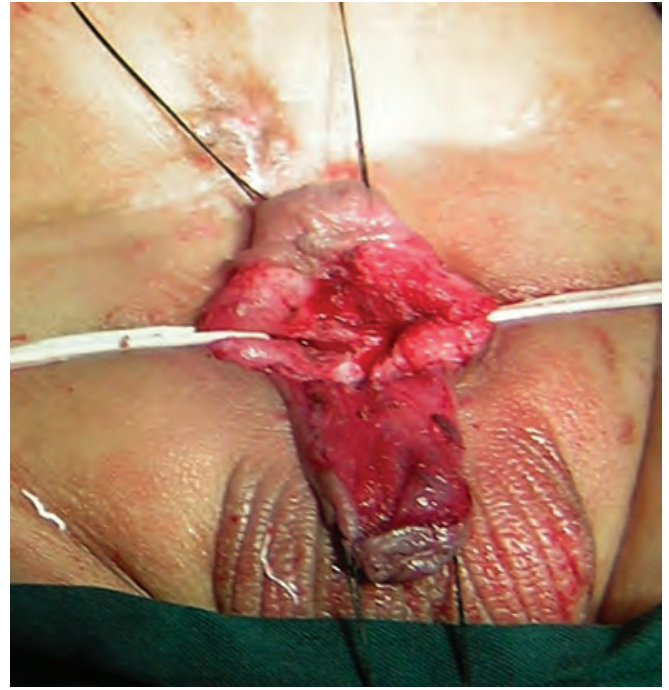

Figure (7): Both corpora dissected and disassembled.

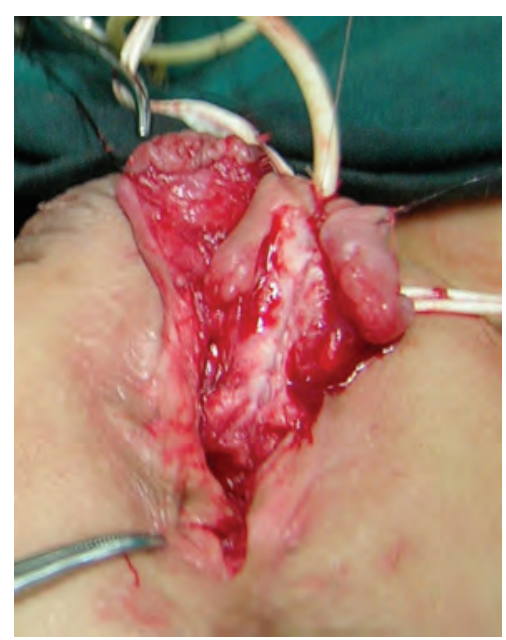

Figure (9): Neo urethra completed using continuous 5/0 PDS sutures.

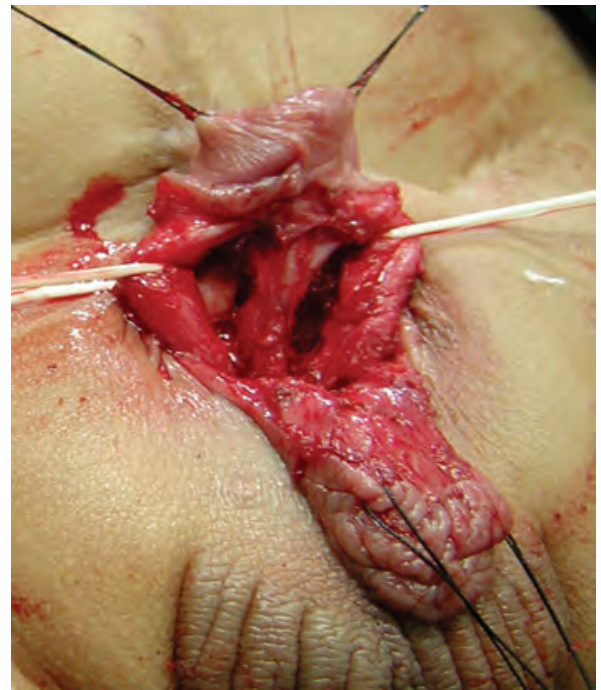

Figure (8): Disassembled 3 components.

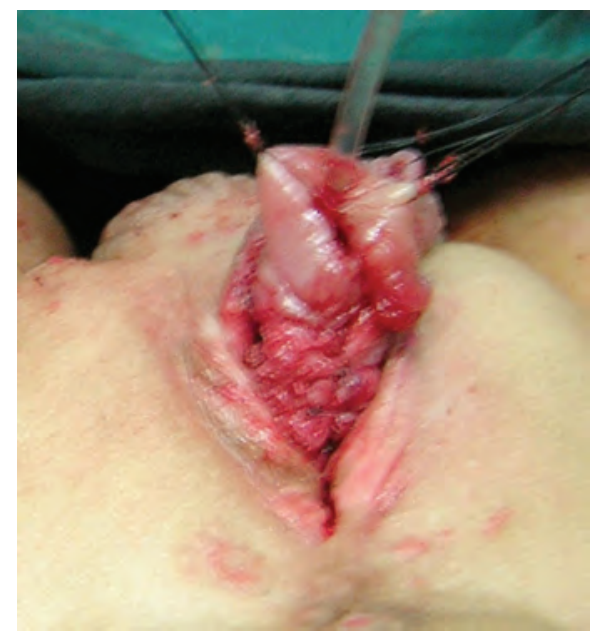

Figure (10): Suturing both corpora over neo urethra.

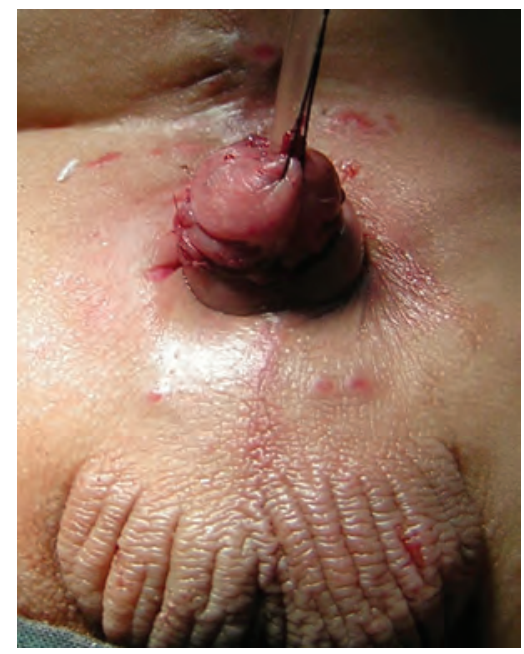

Figure (11): At the end of the operation. 
distal end at the glans, and a tube was created over an 8 French silicon catheter using 5/0 continuous PDS sutures. The corpora were sutured together using interrupted sutures over the ventrally placed neo urethra with internal rotation to prevent occurrence of dorsal chordee.

At the end of operation, a plastic occlusive dressing was applied with urethral catheter kept in place for 7-10 days.

All cases were given IV intra and post operative broad spectrum antibiotics.

As regards post operative complications, only one case showed mild wound infection that was conservatively managed. Another case developed urethro cutaneous fistula, which was surgically treated by simple excision \& closure 6 months later.

Satisfactory reconstruction was obtained in all cases where the penis was achieved with a conical glans, apicoventral meatus, with downward direction on standing.

The mothers of young patients reported straight erections post operatively.

The results of continence could not be evaluated as all cases were too young for such evaluation.

\section{Discussion:}

Epispadias is a rare condition and most commonly described as a part of the bladder exstrophy complex. ${ }^{10}$ This study included 11 cases with epispadias associated with bladder exstrophy in 6 years period. Lottmann et $\mathrm{al}^{3}$ reported 40 cases in 8 years, while Kajbafzadeh et $\mathrm{al}^{4}$ reported 180 cases in 15 years, of them, 75 cases underwent the Cantwell-Ransley operation. Ashraf Hafez et $\mathrm{al}^{11}$ performed 14 post pubertal epispadias cases in 7 years. Their ages ranged from 14 to 34 years.

All our cases had previous first stage bladder exstrophy closure, followed by epispadias repair 1-2 years later. The age incidence of our cases ranged from $17 / 12$ years to 3 years, with a mean of $23 / 12$ years. All were males.

Two cases had a history of associated bilateral congenital inguinal hernias that were repaired in the first stage with closure of bladder exstrophy.

All cases were peno pubic type, with evident dorsal chordee in 3 cases (27.2\%). Hammouda $^{12}$ reported 42 male cases with epispadias in 4 years period. Of them, 29 cases had complete epispadias as a component of bladder exstrophy.

As a rule, all our cases received 3 IM injections of testosterone at monthly intervals before operation, in addition to topical application of testosterone $5 \%$ ointment. This was performed to facilitate penile growth and improving vascularity. Jacob Ben-Cham et al $^{13}$ \& Gearhart \& Jeffs ${ }^{14}$ recommended pre operative administration of testosterone for the same reason.

Lottmann etal ${ }^{3}$ recommended pre operative testosterone administration to increase penile size and blood supply, and hence facilitates its reconstruction. Gearhart ${ }^{15}$ suggested that testosterone application helps to enhance the penile skin, increase vascularity of the urethral plate and soften any area of scarring, which may decrease the incidence of post operative skin and urethral necrosis. ${ }^{15}$

As previously described, all our cases were subjected to the Cantwell-Ransley procedure. Baird et al ${ }^{1}$ performed the same technique to 129 boys, of which 97 had classic bladder exstrophy and 32 had primary complete epispadias.

Artificial erection was performed to all our cases before proceeding to penile disassembly. Saline injection into both corpora separately was performed to detect chordee and assess its direction and extent. We found 3 cases with dorsal chordee that was corrected by excising all fibrous tissue over the corpora that was responsible for angulations.

The neo urethra reached the glans' tip in a normal position without shortening as the distal part of the urethral plate was kept intact. The end result was an apicoventral meatus in a conical glans.

The catheter was kept for 7-10 days then removed. The end result was satisfactory.

Mild post operative edema \& inflammation of the skin was seen in one case, which was conservatively treated and completely resolved. Another case developed 
urethra-cutaneous fistula that was surgically treated by simple excision and closure 6 months later. No urethral stenosis or recurrence was seen among our cases.

Baird et $\mathrm{al}^{1}$ in their large series using the Cantwell-Ransley technique stated that any chosen surgical technique to reconstruct peno pubic epispadias must address four factors: Correction of dorsal chordee, Urethral reconstruction, Glanular reconstruction and Closure of penile skin. They had 25 cases having post operative fistula representing $19.3 \%$, reduced to $15.5 \%$ after 3 months.

Kajbafzadeh et $\mathrm{al}^{4}$ who worked on 180 boys with epispadias performed their new technique in 75 cases. The results were much better than in any of the remaining cases who underwent other techniques. 84\% were regarded as very satisfactory, and a poor outcome requiring minor skin revision, fistula excision or urethral dilatation occurred in only $16 \%$. Fistula occurred in only $4 \%$ and urethral stricture occurred in 5.3\%. They had no case of urethral ballooning or skin dehiscence.

Baird et $\mathrm{al}^{1}$ found that this technique is reliable, and a review of published studies has not demonstrated loss of glans or corporal tissue as reported in other repairs. Surer et al ${ }^{16}$ showed an initial postoperative fistula rate of $23 \%$ reducing to $19 \%$ at 3 months.

Ransley et al ${ }^{17}$, Surer et al ${ }^{16}$ and Mollard et $\mathrm{al}^{18}$ stated that the cosmetic and functional outcomes of Cantwell-Ransley epispadias repair appear to be excellent.

\section{Conclusion}

Any chosen surgical technique to reconstruct peno pubic epispadias must address four factors: Correction of dorsal chordee, urethral reconstruction, glanular reconstruction and closure of penile skin.

The cosmetic and functional outcomes of Cantwell-Ransley epispadias repair appear to be excellent.

\section{Reference}

1- Baird AD, Gearhart JP, Mathews RI: Applications of the modified CantwellRansley epispadias repair in the exstrophyepispadias complex. Journal of Pediatric
Urology 2005; 1: 331-336.

2- Lancaster PAL: Epidemiology of bladder exstrophy: A communication from the international clearinghouse for birth defects monitoring systems. Teratology 1987; 36: 221.

3- Lottmann HB, Yaqouti M, Melin Y: Male Epispadias Repair: Surgical and functional results with the Cantwell-Ransley procedure in 40 patients. The Journal of Urology 1999; 162: 1176-1180.

4- Kajbafzadeh AM, Duffy PG, Ransley PG: The revolution of penile reconstruction in epispadias repair: A report of 180 cases. The Journal of Urology 1995; 154: 858-861.

5- Jr Duckett JW: Epispadias. Urol Clin N Amer 1978; 5: 107.

6- De Sy WA, Oosterlinck W: One-stage hypospadias repair by free full-thickness skin graft and island flap techniques. Urol Clin N Amer 1981; 8: 491.

7- Vyas PR, Roth DR, Perlmutter AD: Experience with free grafts in urethral reconstruction. J Urol 1987; 137: 471.

8- Ransley PG, Duffy PG, Oesch IL, Hoover D: Autologous bladder mucosa graft for urethral substitution. Brit J Urol 1987; 58: 331.

9- Hendren WH, Crooks KK: Tubed free skin graft for construction of male urethra. $J$ Urol 1980; 123: 858.

10- Dominic Frimberger: Diagnosis and management of epispadias. Seminars in Pediatric Surgery, 2011; 20: 85-90.

11- Hafez AT, Helmy T: Complete penile disassembly for epispadias repair in post pubertal patients. Urology, 2011; 78 (6): 1407-1410.

12- Hammouda HM: Results of complete penile disassembly for epispadias repair in 42 patients. The Journal of Urology, 2003; 170: 1963-1965.

13- Ben-Chaim J, Peppas DS, Jeffs RD, Gearhart JP: Complete male epispadias: Genital reconstruction and achieving continence. The Journal of Urology, 1995; 153: 1665-1667.

14- Gearhart JP, Jeffs RD: The use of parenteral testosterone therapy in genital reconstructive surgery. J Urol 1987; 138(2): 1077.

15- Gearhart JP: Evolution of epispadias repair. Timing, techniques and results. J Urol 1998; 160: 177.

16- Surer I, Baker LA, Jeffs RD, Gearhart JP: The modified Cantwell-Ransley repair for exstrophy and epispadias: 10-year experience. J Urol 2000; 164: 1040-1042.

17- Ransley PG, Duffy PG, Wollin M: Bladder 
exstrophy and epispadias. In: Spitz L, Nixon $\mathrm{HH}$, editors. Pediatric Surgery. London: Butterworths; 1988: 620-632.
18- Mollard P, Bassett T, Mure PY: Male epispadias: Experience with 45 cases. $J$ Urol 1998; 160: 55-59. 